\title{
STUDIES OF GENES CONTROLLING SPECIFIC HOST- PARASITE INTERACTIONS IN FLAX AND ITS RUST
}

\section{FINE STRUCTURE ANALYSIS OF THE M GROUP IN THE HOST}

\author{
G. M. E. MAYO and K. W. SHEPHERD \\ Dept. of Genetics and Department of Agronomy, The University of Adelaide, South Australia
}

Received 3.viii.79

\begin{abstract}
Summary
A method used to analyse Flor's $M$ group of genes controlling specific resistance of flax to its rust is described. Using this method, three of seven genes reported so far have been brought together in pairs in coupling. The specific effects of each pair of genes in coupling is a simple sum of each individual gene and identical to the corresponding pair in repulsion, suggesting they are not functionally allelic. Segregational data show that four of these $M$ genes represent closely linked sites separable by recombination and a possible linear order is suggested. Further analysis awaits the availability of appropriate strains of rust to discriminate $M^{2}, M^{5}$ and $M^{6}$ individually and to further distinguish between $M^{1}$ and $M^{4}$.
\end{abstract}

\section{INTRODUCTION}

A STRIKING feature of genes controlling specific host-parasite interactions is the way in which they are often arranged in groups and the question to be resolved is whether members of a group are allelic or separate closely linked genes (Flor, 1956; Saxena and Hooker, 1968; Moseman, 1971).

In flax, at least 29 different dominant genes conferring resistance to a characteristic array of rust strains have been identified (Flor, 1956; Flor and Comstock, 1972; Zimmer and Comstock, 1973; Wicks and Hammond, 1978). They are distributed between five groups, within four of which there are clusters of several closely linked or allelic genes: namely, 13 within the $L$ group, seven in the $M$, three in the $\mathcal{N}$ and five in the $P$ group, whilst only one gene has been identified at Flor's $K$ locus. Within each group these genes behave co-dominantly. Genes drawn from different groups have always shown independent segregation, except between $\mathcal{N}$ and $P$ where recombination fractions varying from 9 to 21 per cent (Flor, 1955; Kerr, 1960; Shepherd, 1963) have been observed. Between genes within one or more of these groups, Shepherd (1963) and Flor (1965) each detected rare recombination.

Shepherd and Mayo (1972) briefly reviewed their results using two such groups of host genes; those at Flor's (1956) $L$ and $M$ loci controlling specific interactions between flax (Linum usitatissimum L.) and its rust (Melampsora lini (Ehrenb.) Lév.). They described a modified cis-trans test for an operational concept of functional allelism between co-dominant genes. Their interim results reported in the same paper showed a difference in behaviour among genes of the $M$ group as distinct from those of the $L$ group: they 
suggested that such divergent behaviour may reflect important differences in their structure.

Since 1972 these investigations have been extended. Recently, a crucial new development showing clearly the compound nature of Flor's $L^{6}$ gene has been observed (Shepherd and Mayo, 1979). This paper reports our methods and most recent results for the $M$ group of genes conferring specific resistance in the host.

\section{Materials AND methods}

Table 1 lists the flax varieties used as parents and the rust strains used to identify the presence of specific $M$ genes in the host plants; their mutual interactions are recorded in the body of the table.

TABLE 1

Interactions between varieties of flax and strains of rust used

\begin{tabular}{|c|c|c|c|c|c|c|c|}
\hline \multicolumn{3}{|l|}{ Host } & \multicolumn{5}{|c|}{ Rust strain } \\
\hline Variety & Genotype & Origin $^{a}$ & 1 & 2 & 3 & 4 & 5 \\
\hline Dakota & $M M$ & C.I. 1071 & - & - & + & + & - \\
\hline Williston Brown & $M^{\mathrm{I}} M^{1}$ & C.I. 803-1 & + & + & + & - & + \\
\hline Cass & $M^{3} M^{3}$ & C.I. 1182 & - & + & - & + & + \\
\hline Victory A & $M^{4} M^{4}$ & C.I. 1170 & + & + & + & - & - \\
\hline $\begin{array}{l}\text { Hoshangabad } \\
\text { (" universal susceptible") }\end{array}$ & $\mathrm{mm}$ & W.I. 89 & + & + & + & + & + \\
\hline
\end{tabular}

a C.I. refers to the accession number of the Division of Cereal Crops and Diseases, United States Department of Agriculture. W.I. refers to the accession number of the Waite Agricultural Research Institute, The University of Adelaide. Stocks of these five rust strains were selected from among numerous progeny derived originally from New Zealand strains 1 to 5 (Cruickshank, 1956).

$$
+=\text { growth of rust; }-=\text { no growth. }
$$

Specific interactions between the two genotypes, the host and its parasite, are reflected in a single phenotype: namely, growth of the parasite, recorded as plus $(+)$ from which the host is inferred to be susceptible (S) and the parasite virulent $(\mathrm{V})$; as distinct from its alternative, no growth (-) on a resistant host $(R)$ infected with an avirulent parasite $(A)$. It is crucial to our analyses that these phenotypes, $(+\mathrm{v}-)$, remain mutually exclusive.

\section{(i) Plant and rust culture}

Host-parasite interaction was tested by inoculating with uredospores plants 5 to $8 \mathrm{~cm}$ high, when the epicotyl had elongated and the first few leaves expanded and separated from the apical bud. When more than one such inoculation was made in sequence, the first strain used was expected to show least growth and/or whose score would permit the greatest number of plants to be discarded thereupon. Subsequent inoculations were made 4 to 6 days after the previous inoculation. Such an interval is sufficient to ensure discrimination between scores of separate inoculations but small enough to permit final inoculations to be made on plants still at a stage of vegetative growth optimal for testing. 
The technique of inoculation depended on its purpose. Where small numbers of plants were inoculated, Flor's (1935) method was used: the tip of each plant was dusted with dry uredospores using a small camel's hair brush, the whole plant exposed to a fine spray of tap water and placed in an atmosphere of 100 per cent humidity for the first 12 hours of incubation. An alternative was to use the hair brush to spread a suspension of uredospores in tap water over the plant leaves and incubate in the same way. Where large numbers, a hundred or more, plants were inoculated, dry uredospores were mixed with an inert talc carrier and sprayed from a gun under steady pressure: a characteristic dose was $0.04 \mathrm{~g}$ of uredospores in $4 \mathrm{~g}$ of talc powder sprayed evenly over approximately 1300 plants distributed over $90,12 \mathrm{~cm}$ pots on a bench area of $90 \times 200 \mathrm{~cm}$.

Mass inoculations involving millions of uredospores or simultaneous maintenance of different strains of actively sporulating rusts called for rigorous precautions to avoid contamination. Each inoculation was performed under complete cover so as to isolate it from all but the target plants: all apparatus after use was thoroughly wetted to destroy stray uredospores. All pots carrying plants inoculated to maintain or test specific rust strains were placed under transparent covers before pustules erupted. These covers enabled several rust strains to be maintained without mutual contamination in the same plant growth cabinet or glasshouse. Large numbers of plants subjected to mass inoculations were isolated in a separate glasshouse, and infected plants or leaves scored and removed before uredospore pustules erupted.

In addition to these precautions, continuous checking for the presence of contaminant rust infections was maintained. The uniform appearance of a dense reaction at the expected eighth to tenth day after inoculation, as against the dispersed appearance both in time and space of a contaminant was nearly always unambiguous. In rare doubtful cases, an excised shoot of the plant was rooted, following Kerr's (1951) technique, and the reaction of the clone retested. Stocks of rust strains in use were tested continuously for trueness to strain on specific differentials. Any stock shown to be contaminated was discarded or re-selected from a single uredospore or an isolated single pustule.

Uredospores of each stock were collected from a population of plants specially grown and inoculated. Sporulating uredospores were collected in isolation, using a room reserved for collecting only from stocks of a given nominated strain, by shaking the infected plants over a large sheet of paper. Uredospores were used fresh from such a collection for definitive tests: when not in active use they were stored in vacuo according to Flor's (1967) technique.

\section{(ii) The breeding programme}

\section{(a) Selection of recombinants among testcross progeny}

The study of recombination within Flor's $M$ locus was initiated by selecting the required rare recombinants among progeny of testcrosses in which two genes, each dominant in their action conferring specific host resistance, were segregating. Table 2 shows the crosses involved in the production of recombinants between any two $M$ genes conferring resistance. These genes, $M^{x}$ and $M^{y}$, differ in the specificity of their action, manifest in the 
range of rust strains to which they confer host resistance. Their recessive alleles, in contrast, are non-specific in that both confer susceptibility to all strains of rust: however, in setting out the breeding programme, it is convenient to separate them formally in the genotype by superscripts indicating the corresponding dominant gene.

Testcross refers to the procedure of crossing $F_{1}$ progeny of these chosen parents with a variety homozygous for the recessive alleles of $M^{x}$ and $M^{y}$, namely, Hoshangabad (see table 1), used in these studies as the so-called " universal susceptible" (Person, 1959). Assuming genes $M^{x}$ and $M^{y}$ can recombine with a frequency $p$ and maintain their specific action after recombination, the expected frequencies of the four testcross progeny phenotypes are set out in table 2. These phenotypes are detected by inoculating

\section{TABLE 2}

Segregation of genes $\mathrm{M}^{\mathrm{x}}$ and $\mathrm{M}^{\mathrm{y}}$ among testcross progeny

Parents

$$
\text { Genotype: } \quad \frac{M^{x} m^{y}}{M^{x} m^{y}} \times \frac{m^{x} M^{y}}{m^{x} M^{y}}
$$

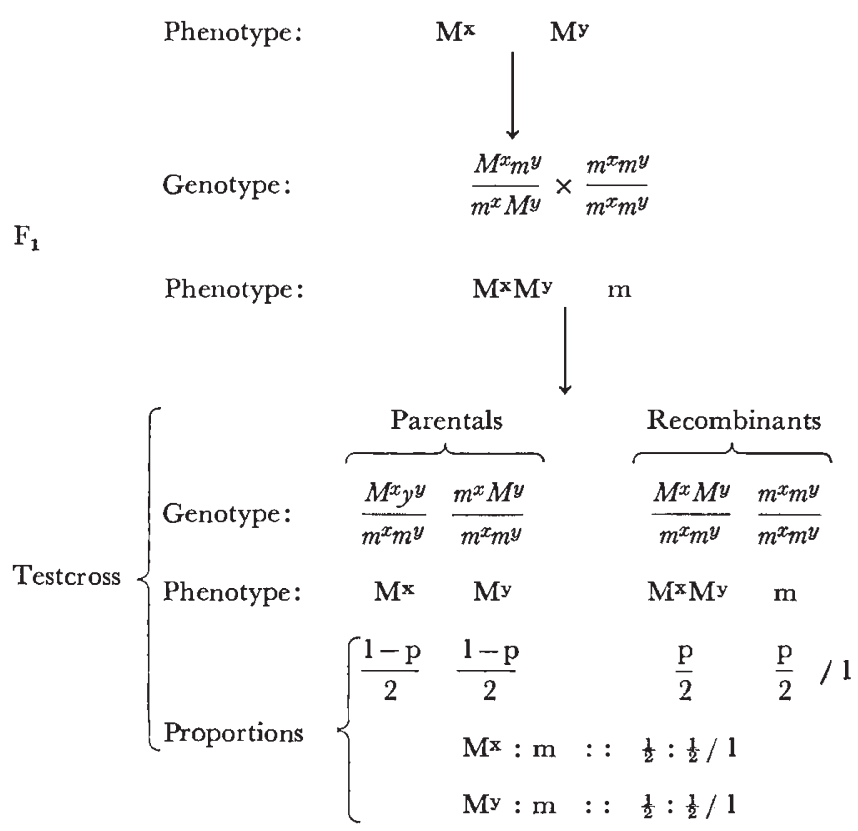

Phenotypes recognised by reaction to selected rust strains

Rust strain

Host phenotype and reaction

\begin{tabular}{|c|c|c|c|c|}
\hline & $M^{x}$ & $\mathrm{M}^{\mathrm{y}}$ & $\mathbf{M}^{x} M^{y}$ & $\mathrm{~m}$ \\
\hline 1 & - & - & - & + \\
\hline 2 & - & + & - & + \\
\hline 3 & + & - & - & + \\
\hline
\end{tabular}


sequentially two strains of rust on each progeny plant, one specific in its avirulence to gene $M^{x}$ and the other to $M^{y}$ (strains 2 and 3 respectively in table 2). In this way, the segregation ratio of each gene among the four phenotypes, as well as their joint segregation, are determined.

Normally a third strain avirulent to both $M^{x}$ and $M^{y}$ is inoculated first in the sequence (strain 1 in table 2): it serves as a partial check of the tests with the other two strains. When strains of types 2 and 3 are not available (see, for example, Saxena and Hooker, 1968), the use of strain 1 enables only the double recessive recombinant to be detected.

\section{(b) $F_{2}$ analysis of selected recombinants}

Once a double dominant recombinant heterozygote in coupling $\left(M^{x} M^{y}\right)$ $m^{x} m^{y}$ in table 2) has been selected from among testcross progeny, natural self-pollination in flax may be exploited to produce large families of segregating progeny. In coupling, when the recombination value $p$ is very small it is estimated with practically the same efficiency from such " $F_{2}$ 's" as from backcrosses per segregant scored (Mather, 1951). Table 3 sets out these expectations: when $p$ is very small, that part of expectations which are functions of $p^{2}$ may be ignored.

Such progeny provide not only a further efficient estimate of recombination between pairs of dominant genes, such as $M^{x}$ and $M^{y}$, but they also provide independent evidence that genetical recombination is involved since the recombinants should have the same specific reactions to strains 1,2 and 3 as the parental types of the original testcross. Again, segregations of the individual genes are tested simultaneously.

However, without interfering with this convenient and efficient estimation of recombination, a chosen third gene, e.g. $M^{z}$, can be introduced in repulsion to $M^{x}$ and $M^{y}$ so that the hitherto pairwise analysis is extended to an orthogonal analysis of three such genes, provided $M^{z}$ maintains its specificity in the presence of $M^{x}$ and $M^{y}$.

Table 3 illustrates how the double dominant heterozygote in coupling, selected from among the testcross progeny may be crossed directly to a variety carrying the chosen third gene, $M^{z}$. The $M^{z}$ gene throughout table 3 is shown genotypically in parenthesis, whilst it is not being identified phenotypically. None of the rust strains, 1, 2 or 3, used during these stages of the analysis are avirulent to $M^{z}$.

The presence of a third gene enables a further crucial development of the analysis. Plants with the recombinant phenotypes $M^{x}$ and $M^{y}$ in table 3 are allowed to self-pollinate, and in the families raised from the resulting seed, $M^{z}$ is identified by inoculating with rust strain 4 specifically avirulent to it. According to the pattern of these segregations, a choice between three possible locations of the third gene with respect to the original pair is made. Table 4 sets out the patterns of segregation to be expected from the three possible locations.

If $M^{z}$ is distal to $M^{y}$ (order $I$, table 4) and if recombination between $M^{x}$ and $M^{y}$ is reciprocal the recombinant gamete $M^{x} m^{y}$ should also carry $M^{z}$. The reciprocal recombinant gamete should then be $m^{x} M^{y} m^{z}$ giving the genotypes $M^{x} m^{y} M^{z} / m^{x} m^{y} M^{z}$ and $m^{x} M^{y} m^{z} / m^{x} m^{y} M^{z}$ respectively. Selfing of such genotypes will give families which show no segregation in the former but in the latter, one-quarter of the progeny will be homozygous $m^{z}$. By 
TABLE 3

Segregation of genes $\mathrm{M}^{\mathrm{x}}, \mathrm{M}^{\mathrm{y}}$ and $\mathrm{M}^{\mathrm{z}}$ among $F_{2}$ progeny

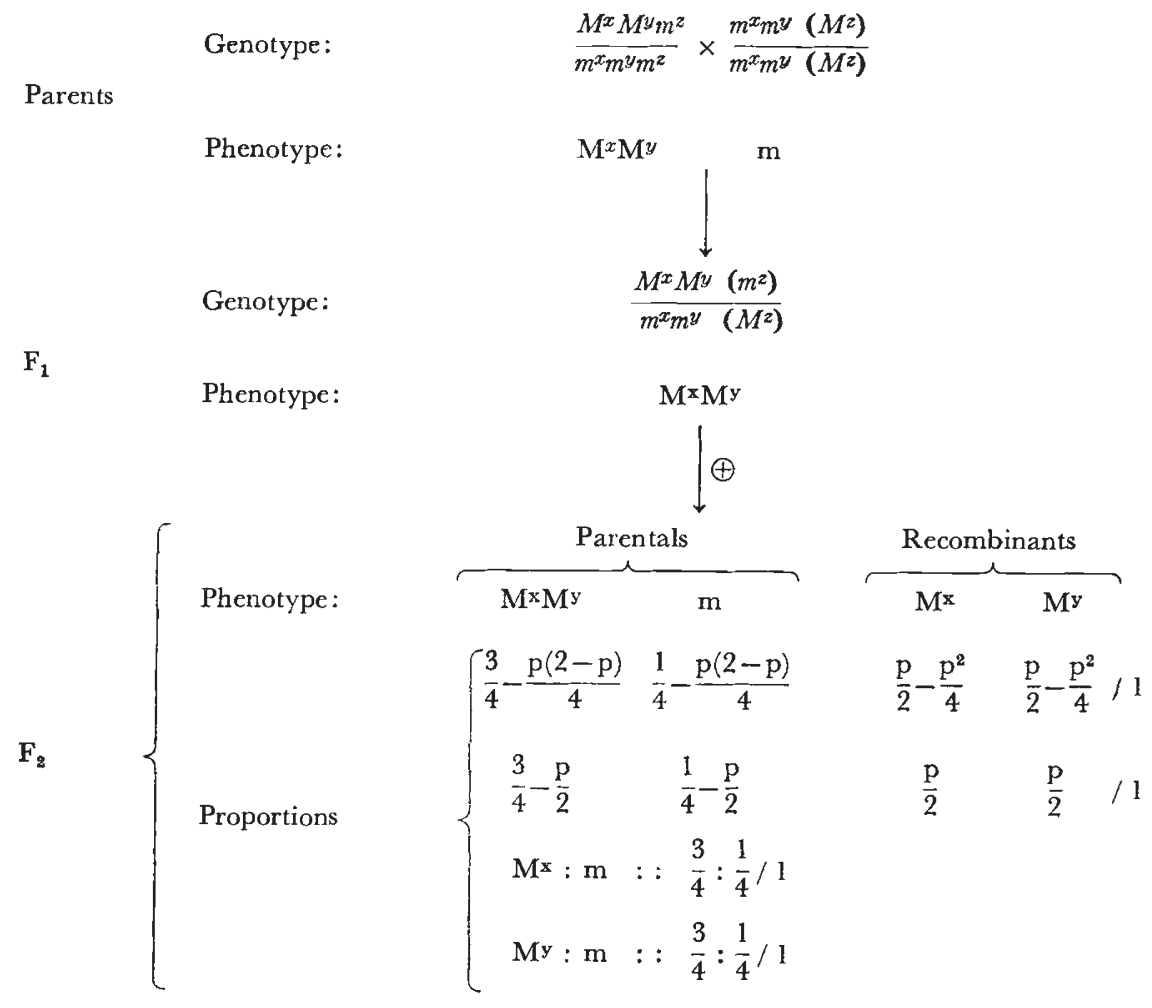

Reaction to selected rust strains

Rust strain

Host

\begin{tabular}{|c|c|c|c|}
\hline \multicolumn{4}{|c|}{ Phenotype } \\
\hline $\mathrm{M}^{\mathrm{x}} \mathrm{M}^{\mathrm{y}}$ & $\mathrm{m}$ & $M^{x}$ & $\mathrm{M}^{\mathrm{y}}$ \\
\hline- & + & - & - \\
\hline- & + & - & + \\
\hline- & + & + & - \\
\hline
\end{tabular}

\begin{tabular}{|c|c|c|}
\hline \multicolumn{3}{|c|}{ Gene } \\
\hline$M^{x}$ & $M^{y}$ & $\left|M^{z}\right\rangle$ \\
\hline- & - & +1 \\
\hline- & + & + \\
\hline+ & - & $1+1$ \\
\hline
\end{tabular}

At this stage, order of genes is unknown and is entered arbitrarily.

( ) indicates no specific resistance to strains 1,2 and 3.

$+=$ growth of rust: $-=$ no growth.

$\mathrm{p}=$ recombination fraction between $M^{x}$ and $M^{\nu}$.

$\oplus=$ selfing.

raising and testing at least 16 progeny in each family with rust strain 4, these genotypes may be distinguished at a 99 per cent level of certainty.

If, however, the $M^{z}$ gene is proximal to $M^{x}$ and $M^{y}$ (order II, table 4), by the same argument, the pattern of segregation of $M^{z}$ among the progeny of the selected recombinants will be reversed: recombinant $M^{x}$ will give rise to segregating progeny but $M^{y}$ will fail to do so. 


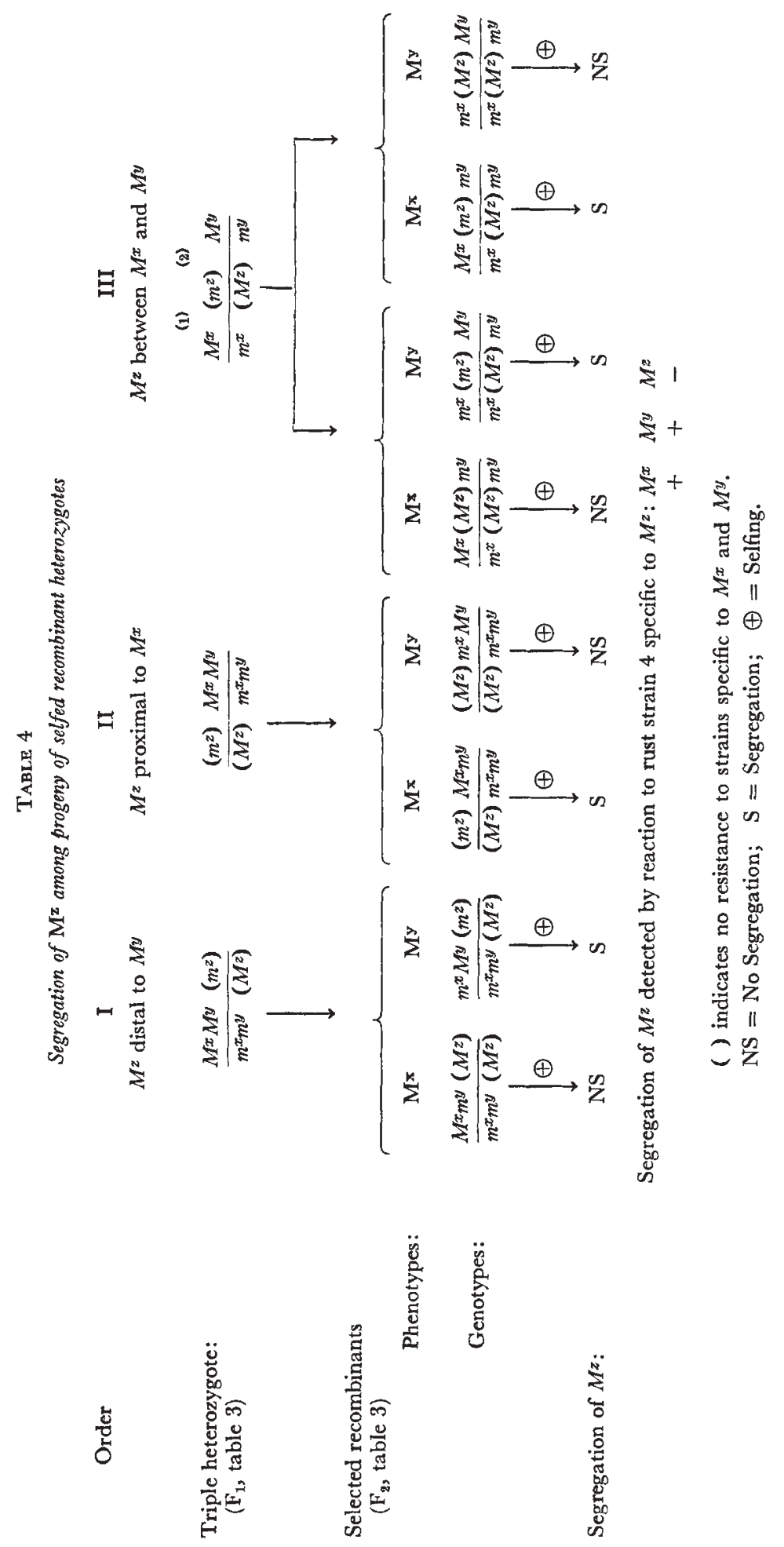


Finally, should the gene $M^{z}$ lie between $M^{x}$ and $M^{y}$, both patterns of segregation will be observed (order III, table 4), since there are two alternative regions of recombination that could give rise to the selected recombinants. If recombination occurs between the genes $M^{x}$ and $M^{z}$ (region (1), order III, table 4), recombinant $\mathrm{Mx}$ will be homozygous and recombinant $M^{y}$ heterozygous with respect to $M^{z}$ : recombination between $M^{z}$ and $M^{y}$ (region (2)) will lead to a reversed pattern of segregation. Thus, selffertilization of both $\mathbf{M}^{\mathrm{x}}$ and $\mathbf{M}^{\mathrm{y}}$ plants, will give rise to families showing either segregation or no segregation with respect to $M^{z}$.

This breeding programme is self-generative in providing new combinations of dominant genes in coupling within the series of three. It is apparent from table 4 that no matter which of the three orders is correct, at least either $M^{x} M^{z}$ or $M^{y} M^{z}$ become available to repeat a further cycle of the breeding procedure to determine, in turn, the location of $M^{x}$ with respect to $M^{y}$ and $M^{z}$, or $M^{y}$ with respect to $M^{x}$ and $M^{z}$. By performing the cycle of breeding, if necessary, up to three times an orthogonal determination of the order of three such genes should be forthcoming. By crossing pairs of these $M$ genes out to further members of the series, this analysis can be extended. Such a procedure also provides genotypes made up of different combinations of genes, whose phenotypes may be studied and interpreted in terms of our modified cis-trans test (Shepherd and Mayo, 1972).

\section{RESUlts}

Families of testcross progeny were derived from $16 \mathrm{~F}_{\mathbf{1}}$ plants, themselves progeny of reciprocal crosses between the original parents, Dakota $(M M)$ and Cass $\left(M^{3} M^{3}\right)$. These $\mathrm{F}_{1}$ plants were used as female parents in the subsequent cross with the " universal susceptible" parent. The families of testcross progeny so generated segregated homogeneously and are consequently pooled in table 5 .

Rust strains 2 and 3 in table 1 were specific in their recognition of gene $M$ in Dakota and $M^{3}$ in Cass respectively: these genes conformed to a Mendelian monohybrid segregation, $M$ with respect to its allele $m$ and $M^{3}$ with respect to $m^{3}$.

These dominant genes were detected unambiguously throughout these and subsequent segregations, not only by their interaction with specific rust strains, but also by their characteristic phenotypes: $M$ caused small necrotic flecks whilst infected leaves carrying only $M^{3}$ had larger necrotic areas with surrounding chlorosis.

One of the double dominant recombinant progeny of the testcross was allowed to mature and self-pollinate in strict isolation under conditions designed to encourage maximum seed production (table 5). A random sample of 50 progeny was tested against rust strain 1, avirulent to either $M$ or $M^{3}$ : 37 were resistant and, of these, 24 out of a sample of 29 tested proved to be heterozygous for $M$ and $M^{3}$.

A random sample of ten of those heterozygous for $M$ and $M^{3}$ was used to raise large numbers of segregating progeny and these were screened for recombinants between $M$ and $M^{3}$ (group 1, table 6). Two of the five plants judged homozygous for $M$ and $M^{3}$ (one producing white and the other blue flowers) were selected and crossed to Victory $\mathrm{A}\left(M^{4}\right)$ and Williston Brown $\left(M^{1}\right)$. Large numbers of $\mathrm{F}_{2}$ seedlings of these outcrosses were also raised 


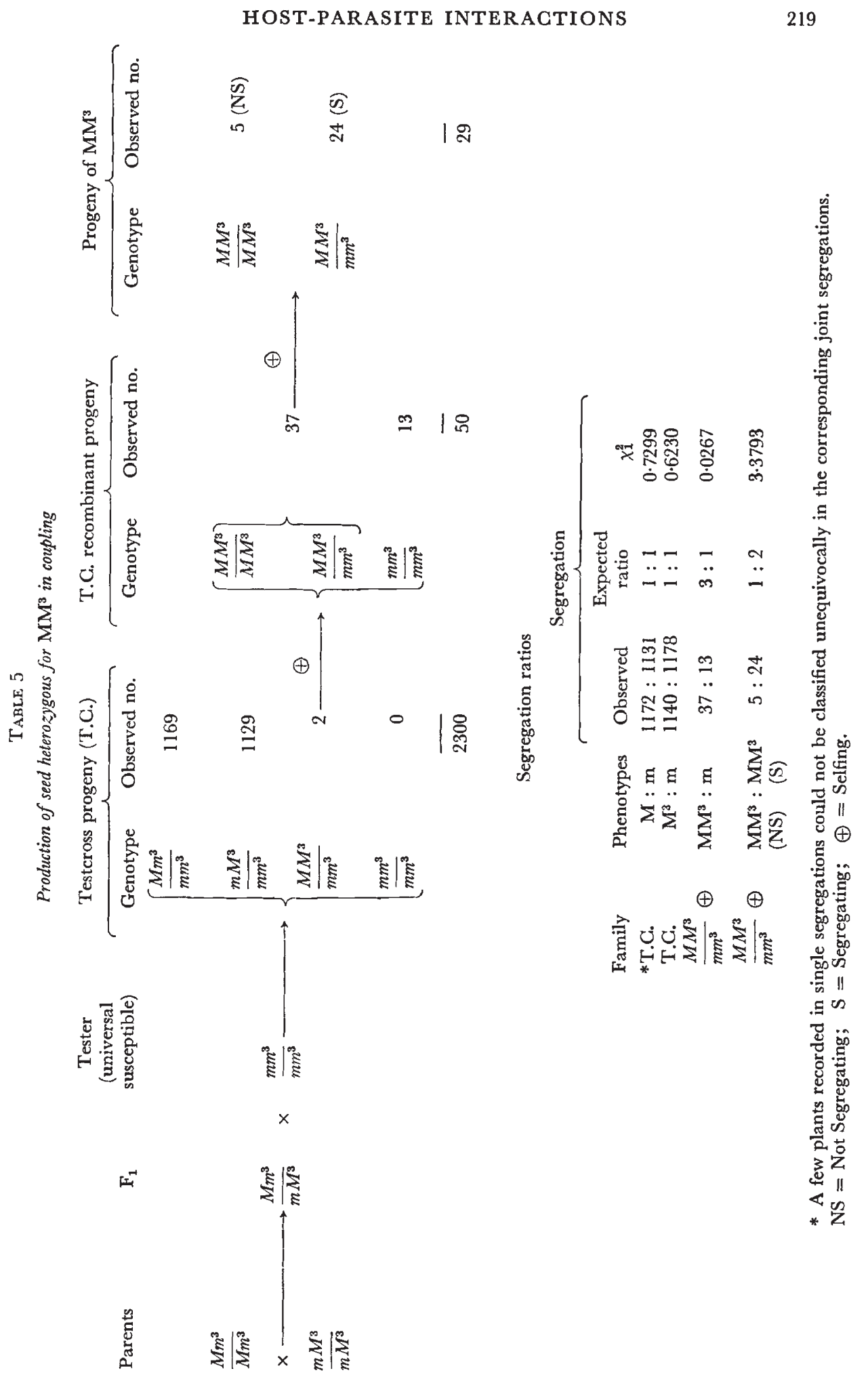




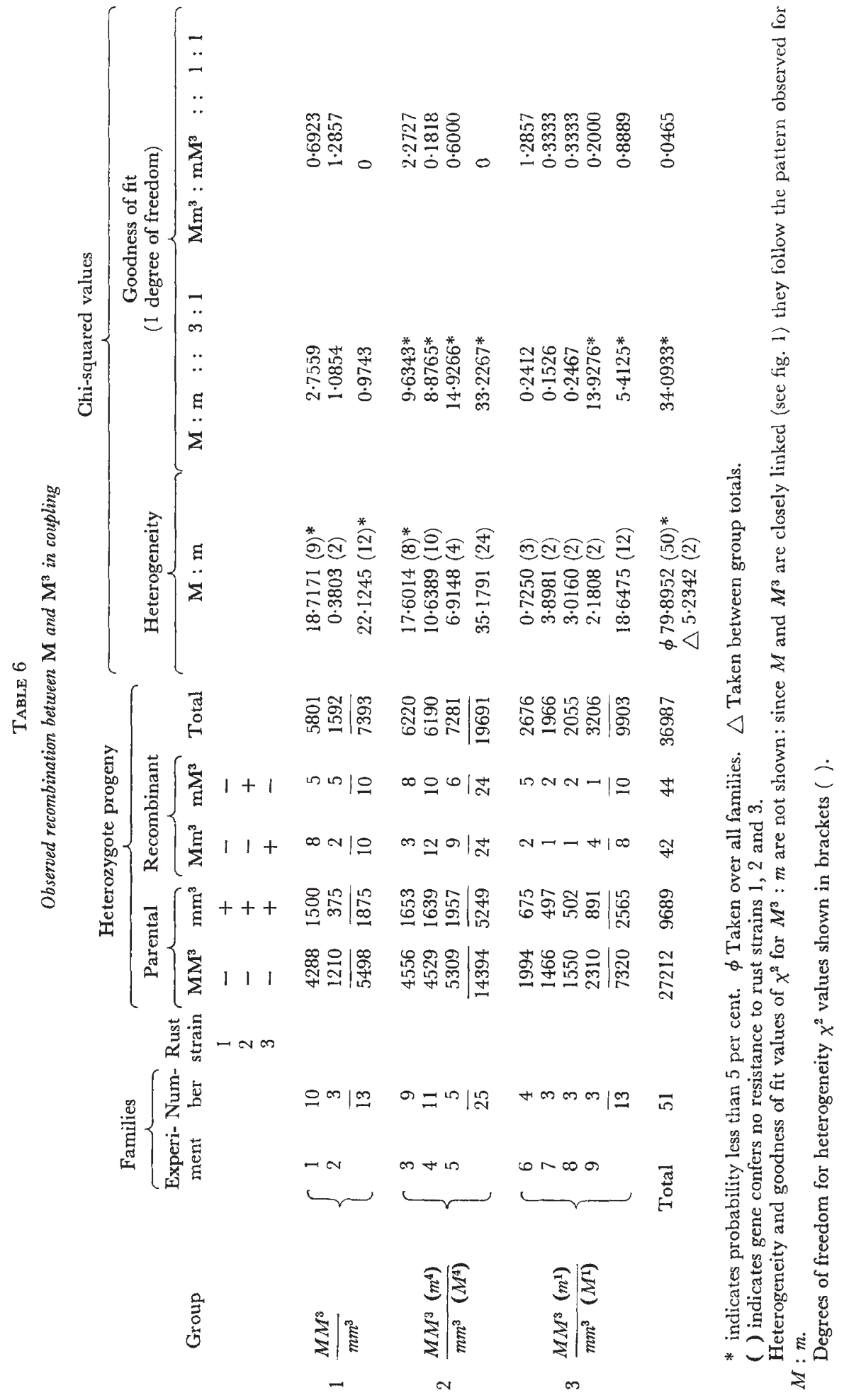


and screened for recombinants between $M$ and $M^{3}$, whilst $M^{4}$ (group 2, table 6) and $M^{1}$ (group 3, table 6) respectively were allowed to segregate unrecognised.

Nearly 37,000 segregants were observed among these various $F_{2}$ progeny, drawn from 51 families and involving four $M$ genes. Results and $\chi^{2}$ tests are shown in table 6 . Eighty-six recombinants, segregating consistently in agreement with a $1: 1$ ratio of reciprocal products from double heterozygotes for $M$ and $M^{3}$ in coupling, were recovered against a background in which the individual gene pairs, $M: m$ and $M^{3}: m^{3}$ each approximated to simple Mendelian segregations. These latter segregations were not, however, entirely homogeneous between families, specifically in experiments 1 and 3 , reflecting, perhaps, some variation in gametogenesis in the different genotypes generating the progeny. In four of the nine experiments, a significant excess of homozygous recessive genotypes was observed among the segregants, three in families carrying $M^{4}$. The reason for these deviations is not clear. They are unlikely to be due to effects directly ascribable to changes in environment since all plants in table 6 were grown under conditions optimal for expression of the genes $M$ and $M^{3}$ and, in particular, the $\mathrm{F}_{1}$ and the $M M^{3} / \mathrm{mm}$ recombinant plants used as parents were grown at the same time of year with near to identical conditions. Subsequently progeny tests of recombinants recovered again confirmed that both $M$ and $M^{3}$ segregated in a simple Mendelian fashion.

Each recombinant recovered was allowed to self-pollinate. From among the 48 recombinants recovered in experiments 3 to 5,23 were available to raise families of progeny and tested for the presence of $M^{4}$. Similarly, 13 families of progeny were available from among 18 recombinants recovered in experiments 6 to 9 and tested for the presence of $M^{1}$.

Amongst the 13 families from $\mathrm{Mm}^{3}$ recombinants only one showed segregation for $M^{4}$ (see table 7). However, $M^{4}$ segregated in all 10 families from $\mathrm{mM}^{3}$ recombinants. These observations suggested tentatively that $M^{4}$ lies between $M$ and $M^{3}$ with $M^{4}$ closer to $M^{3}$ since the frequency of recombination between $M$ and $M^{4}$ is much greater than that between $M^{4}$ and $M^{3}$. In all four families from $\mathrm{Mm}^{3}$ recombinants, $M^{1}$ for its part, failed to segregate whilst it segregated in all nine families from $\mathrm{mM}^{3}$ recombinants. Again a tentative suggestion that $M^{1}$ is distal to $M$ and $M^{3}$ is made.

\section{TABLE 7}

Segregation of $\mathrm{M}^{4}$ and $\mathrm{M}^{1}$ among progeny of recombinants $\mathrm{Mm}^{3}$ and $\mathrm{mM}^{3}$

\begin{tabular}{|c|c|c|c|c|c|}
\hline \multirow{3}{*}{\multicolumn{2}{|c|}{ Recombinant }} & \multicolumn{3}{|c|}{ Number of families } & \\
\hline & & \multicolumn{2}{|c|}{ Segregating } & \multirow[t]{2}{*}{ Total } & \multirow[b]{2}{*}{ Inferred order } \\
\hline & & Yes & No & & \\
\hline \multirow{3}{*}{$M^{4}$} & $\mathrm{Mm}^{3}$ & 1 & 12 & \multirow{3}{*}{23} & \multirow{3}{*}{$M^{4}$ between $M$ and $M^{3}$} \\
\hline & & & & & \\
\hline & $\mathrm{mM}^{3}$ & 10 & 0 & & \\
\hline \multirow[b]{2}{*}{$M^{1}$} & $\mathrm{Mm}^{3}$ & 0 & 4 & \multirow[b]{2}{*}{13} & \multirow[b]{2}{*}{$M^{1}$ distal to $M$ and $M^{3}$} \\
\hline & $\mathrm{mM}^{3}$ & 9 & n & & \\
\hline
\end{tabular}

Failure of families to show segregation was determined at a probability of 0.01 or less. 


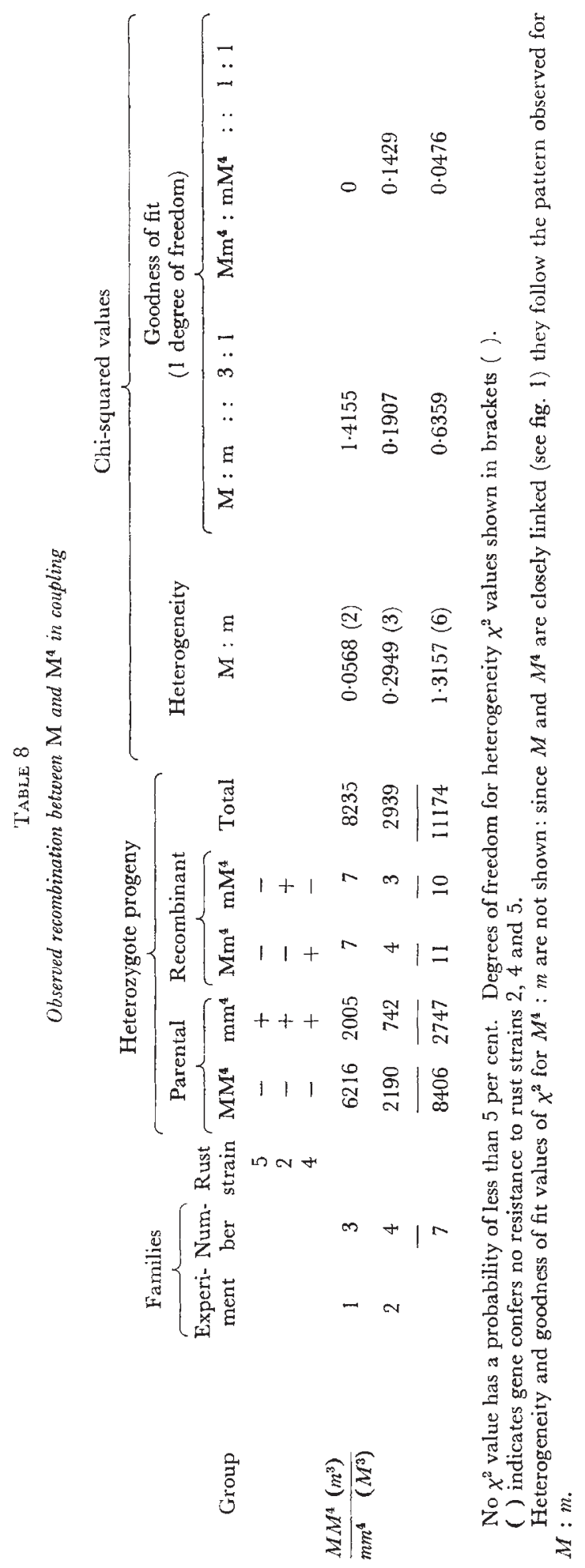


TABLE 9

Segregation of $\mathbf{M}^{3}$ among progeny of recombinants $\mathrm{Mm}^{4}$ and $m M^{4}$

\begin{tabular}{|c|c|c|c|c|}
\hline \multirow[b]{3}{*}{ Recombinant } & \multicolumn{3}{|c|}{ Number of families } & \\
\hline & \multicolumn{2}{|c|}{ Segregating } & \multirow[t]{2}{*}{ Total } & \\
\hline & Yes & No & & Inferred order \\
\hline $\mathrm{Mm}^{4}$ & 10 & 0 & \multirow{2}{*}{20} & \multirow{2}{*}{$M^{3}$ distal to $M$ and $M^{4}$} \\
\hline $\mathrm{mM}^{4}$ & 0 & 10 & & \\
\hline
\end{tabular}

Failure of families to show segregation was determined at a probability of 0.01 or less.

$M M^{4}$ in coupling was recovered from among progeny families from $M^{3}$ in which $M^{4}$ failed to segregate. Individuals with this genotype were crossed with Cass $\left(M^{3}\right)$ to produce the genotype $M M^{4} \mathrm{~m}^{3} / \mathrm{mm}^{4} M^{3}$ which was used, in turn, to initiate a further cycle of the analysis (table 8 ). The progeny from selfing such individuals were screened for recombinants between $M$ and $M^{4}$, keeping $M^{3}$ unrecognised. Table 8 shows that from over eleven thousand plants distributed among seven families, 21 recombinants, segregating in a 1:1 ratio of reciprocal products, were recovered against a homogeneous background in which $M: m$ and $M^{4}: m^{4}$ each segregated in agreement with the expected $3: 1$ Mendelian ratios. Ten progeny families of each recombinant type were raised subsequently (table 9 ). All 10 derived from $\mathrm{Mm}^{4}$ segregated with respect to $M^{3}$ whilst all those from $\mathrm{mM}^{4}$ failed to do so. These results conform to the model in which $M^{3}$ is distal to $M$ and $M^{4}$. This result together with those of $M^{4}$ and $M^{1}$ with respect to $M$ and $M^{3}$ (table 7) suggest a consistent gene order (fig. 1). However, this

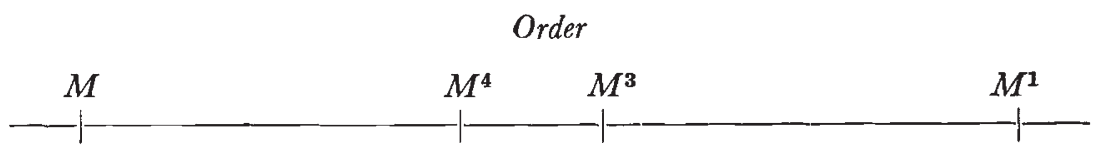

$$
\begin{array}{cr}
\multicolumn{2}{c}{\text { Recombination fraction }} \\
\text { Method of estimation } \\
M-M^{3}
\end{array}
$$

Maximum likelihood

Testcross and " $F_{2}$ " progeny $\quad 0.24 \pm 0.03 \%$ combined

Testcross progeny

" $\mathrm{F}_{2}$ " progeny

$0.09 \pm 0.06 \%$

$0 \cdot 24 \pm 0.02 \% \quad 0 \cdot 19 \pm 0 \cdot 04 \%$

Stevens' table

Mean

Testcross progeny $\quad 0.09 \%$

" $F_{2}$ " progeny

Limits $(\mathbf{P}=0.05)$

Testcross progeny $\quad 0.01$ to $0.31 \%$

" $\mathrm{F}_{2}$ " progeny $\quad 0.20$ to $0.30 \%$

FIG. 1.-Genetic map of $M$ genes. 
consistency is not reflected in estimates of genetic map distance as represented by the recombination fractions between $M$ and $M^{3}$ and $M$ and $M^{4}$.

These estimates are tabulated in fig. 1. Using the method of maximum likelihood (Mather, 1951), there was heterogeneity between testcrosses and " $\mathrm{F}_{2}$ " progeny at a 5 per cent level of probability $\left(\chi_{1}^{2}=5.9234\right)$ in the combined estimation. These estimates take no account of skew inevitable in distributions of such rare recombination. Even so, estimates based on Poisson distributions (Stevens, 1942) show virtually total overlap in their limits $(\mathrm{P}=0.05)$ between $M$ and $M^{3}$ and $M$ and $M^{4}$. No estimate can be made at present between $M^{1}$ and any of the other three $M$ genes.

\section{Discussion}

This analysis of a group of genes concerned with specific host resistance is based inter alia on several assumptions that need closer examination, namely:

1. Each parental host variety under analysis possesses a different single dominant gene controlling resistance (table 1).

2. Notwithstanding their low frequency, non-parental products of gametogenesis throughout this analysis are the result of reciprocal recombination.

3. Recombinants, selected and subsequently progeny-tested with respect to a third $M$ gene, are themselves the result of unions of recombinant and corresponding parental, double recessive, gametes (table 4).

4. None of these recombinant gametes are the product of double recombination.

With respect to the first assumption, although $M$ genes in this analysis behave rigorously as units of segregation, they may be functionally more than one gene. Person (1959) suggested that, within the range of rust strains by which Abyssinian and Leona are classified by Flor (1956) to possess one gene each, $P^{2}$ and $P^{3}$ respectively, there are, in fact, two and three such genes respectively. However, Lawrence (1977) produced clear evidence inconsistent with such a hypothesis. Nevertheless, Shepherd and Mayo (1979) have demonstrated the compound nature of Flor's $L^{6}$. Also there is suggestive evidence (Lawrence et al., 1979) that Victory A and Towner, assigned by Flor (1956) the single genes $M^{4}$ and $L^{8}$ respectively, may possess $M^{1}$ and $M^{4}$ and $L^{8}$ and $M^{4}$ respectively.

The question therefore arises as to whether the use of a variety, such as Victory A, possessing more than one $M$ gene, will thereby invalidate the analysis. In fact, it will proceed conventionally provided the two or more genes segregate as a single unit and maintain their original combined interaction with the strains of rust used. However, should new strains that distinguish between two or more of these genes be introduced, it could detect any products of rare recombination that maintain trueness to type of the component genes. Thus, this analysis is a continuing one that can be refined not only by screening larger numbers of segregants but also by using strains of rust able to discriminate new combinations of host genes.

The low frequency of the non-parental products of gametogenesis might suggest that they are the result of mutations. Among 23 tested recombinants between $M$ and $M^{3}$ only one was observed to occur between $M^{4}$ and $M^{3}$ in 
region (2) (tables 4 and 7 ). Taking this and the lower limit, at $\mathrm{P}=0.05$, of the estimate of the recombination fraction between $M$ and $M^{3}$ as 0.0001 (fig. 1), then the lowest value possible for $M^{3}$ and $M^{4}$ would be $\frac{1}{23} \times 0 \cdot 0001$, that is, $4 \times 10^{-6}$, well within the order of the expected general mutation rate. However, this represents an extreme lower limit and is of a different magnitude from the direct estimate of $1 \cdot 2 \times 10^{-3}(0.12$ per cent in fig. 1$)$. Moreover, the fact that the new combinations recovered are reciprocal suggests that they arose by recombination rather than mutation.

In the absence of flanking marker genes the distinction between reciprocal recombination and non-reciprocal recombination or conversion is impossible. If all of the observed recombinants are the result of conversion of either of the two genes in any given test and if this can occur in either direction, i.e. $M \rightleftharpoons m$, and at either site, then it is not possible to distinguish the three orders as set out in table 4 . The recovery of statistically equal numbers of the two possible recombinants could simply mean that conversion is equally likely from $M \rightarrow m$ and vice versa. Only if recombination is between non-allelic genes can it be presumed to be reciprocal in the sense that both products arise from a single recombination event, and only then can the order of the $M$ genes be determined. However, the observation that the phenotype of any two $M$ genes in coupling is the same as in repulsion suggests that the $M$ genes are not functionally allelic and that the $M$ genes represent a series of closely linked genes, functionally distinct but probably functionally similar. If this is the case, then it is more likely that the recombination between them is reciprocal and the analysis summarised in Figure 1 is valid.

With respect to the third assumption, should by rare chance any other than the common gamete, $\mathrm{mm}^{3} \mathrm{M}^{4}$ in Order I or its equivalent in the alternative models (table 4), pair with the recombinant gametes, the resultant genotype would fail to be selected as a recombinant or it would lead to disturbances of the expected segregation patterns, easily confirmed by simple additional progeny tests. No such disturbances were observed.

The probability of double recombinants occurring in a single gamete will be the product of the probabilities of two single recombinants, of the order of $10^{-5}$. In two of the orders, I and II in table 4, but not the third, such an unlikely selection of a double recombinant would present subsequent misleading segregation patterns of the third $M$ gene. However, once each of the genes in the series of three has been located, in turn, as the third gene with respect to the other two, any anomalies arising from selection of a double recombinant will produce one of the segregation patterns incompatible to the other two. Where the incompatibility stems from one aberrant family, the possible presence of a double recombinant is reinforced, since the recurrence of two double recombinants in a single series is so remote that it may be ignored. Again, further progeny testing should resolve the situation.

To sum up, rigorous application of relatively simple routine tests ensured the validity of the qualitative determination of gene order: our results, so far, conform to the interpretation of fig. 1.

However, among seven $M$ genes that have been identified (Flor, 1956; Zimmer and Comstock, 1973), providing a potential of 21 pairs of $M$ genes, only 4 pairs have been fully or partially analysed. Further progress is dependent on the availability of rust strains providing satisfactory differential 
specificity to the $M$ genes, $M^{2}, M^{5}$ and $M^{6}$. In addition, a strain that will clearly differentiate $M^{1}$ and $M^{4}$ is needed to resolve recent results suggesting $M^{1}$ and $M^{4}$ are both present in Victory A (Lawrence et al., 1979).

Since all pairs of genes in coupling, $M M^{3}, M M^{4}$ and $M^{4} M^{3}$ so far recovered show identical phenotypes to that of their respective genotypes in repulsion, further support is provided for Shepherd and Mayo's (1972) suggestion, based on their modified cis-trans test, that $M$ genes are situated at separate closely linked loci and function independently of each other.

Finally, multiple heterozygotes containing two $M$ genes in coupling in each strand may be used to breed genotypes with three and, ultimately, more than three $M$ genes together in coupling, always providing appropriate strains of rust are available. Construction of these complex genotypes would provide an extended basis for applying the modified cis-trans test as well as having considerable practical implications in breeding for disease resistance.

Although genes, simply inherited and conferring specific resistance to disease, have been used extensively in commercial crops, very little is known about their structure. One impediment to such studies has been the difficulty in generating large numbers of testcross progeny in crops that are selfpollinated. However, where ingenuity can be invoked to select for the first recombinant, for example, in the use of male sterility to enforce outcrossing without hand manipulation, the $F_{2}$ analysis applied here to flax should provide a useful tool in studying the nature of these genes over a much broader field.

So far, similar analyses of genes conferring specific resistance have been rare. An outstanding exception is the study of the $R p_{1}$ locus reported by Saxena and Hooker (1968) and Hooker and Saxena (1971). Using seven large testcross families, ranging in size from 4170 to 19641 individuals, they tested seven $R p_{1}$ genes for recombination in pairwise crosses and in all cases recombinants were recovered. In four of the families only one of the two recombinants was scored, but in the three largest families both recombinants were identified and since these occurred with approximately equal frequencies they assumed recombination was reciprocal although conversion could not be excluded. From their results they suggested a tentative linear map in which the gene $R p_{1}{ }^{c}$ lies between $R p_{1}{ }^{a}$ and $R p_{1}{ }^{k}$. However, since flanking markers were not used in scoring the testcross segregations and the 95 per cent confidence limits show almost complete overlap in estimates of the relative location of the three alleles, the benefit of attempting a qualitative estimate of order, using the $\mathrm{F}_{2}$ method of analysis, is apparent.

\footnotetext{
Acknowledgments.-We express our appreciation to the Australian Research Grants Committee for financial support, to Heather Stock and Margaret Lamey for technical assistance of high calibre, and to our colleagues, especially Dr Jean Mayo, for valued discussions.
}

\section{REFERENGES}

CRUICKSHANK, I. A. M. 1956. A further note on the physiologic specialisation in Melampsora lini (Enrenb.) Lév. in New Zealand. New Zealand 7. Sci. Tech. B, 38, 85-88.

FLoR, H. H. 1935. Physiologic specialisation of Melampsora lini on Linum usitatissimum. U.S.D.A. 7. Agric. Res., 51, 819-837.

FLOR, H. H. 1955. Host-parasite interaction in flax rust-its genetics and other implications. Phytopath., 45, 680-685. 
FLOR, н. H. 1956. The complementary genic systems in flax and flax rust. Adv. Genet., 8, 29-54.

FLOR, H. H. 1965. Tests for allelism of rust-resistance genes in flax. Crop Sci., 5, 415-418. FLOR, H. H. 1967. Preservation of urediospores of Melampsora lini. Phytopath., 57, 320-321. FLOR, H. H., AND COMSTOCK, v. E. 1972. Identification of rust conditioning genes in flax cultures. Crop Sci., 12, 800-804.

HOOKER, A. L., AND SAXENA, K. м. s. 1971. Genetics of disease resistance in plants. Ann. Rev. of Genetics, 5, 407-424.

KERR, H. B. 1951. The use of excised shoots in linseed investigations. Proc. Linnean Soc. of N.S.W., lxxvi, 183-187.

KERR, H. B. 1960. The inheritance of resistance of Linum usitatissimum L. to the Australian Melampsora lini (Pers.) Lév. race complex. Proc. Linnean Soc. of N.S.W., lxxxv, 273-321. LAWRENCE, G. J. 1977. Genetics of pathogenicity in flax rust. Ph.D. Thesis, The University of Adelaide.

LAWRENGE, G. J., MAYO, G. M. E., AND SHEPHERD, K. W. 1979. Interactions between genes controlling pathogenicity. Phytopath. (in press).

MATHER, к. 1951. The Measurement of Linkage in Heredity, 2nd Edition Rev. and Enlarged. 149 pp. Methuen and Co. Ltd., London.

moseman, J. G. 1971. Genes for specific resistance: powdery mildew of barley. Phytopath., $61,617-626$.

PERsON, c. o. 1959. Gene-for-gene relationships in host-parasite systems. Canad. F. Bot., 37, $1101-1130$.

SAXENA, K. M., AND HOOKER, A. L. 1968. On the structure of a gene for disease resistance in Maize. P.N.A.S., 61, 1300-1305.

SHEPHerd, K. w. 1963. Genetics of host-pathogen interactions. Ph.D. Thesis, The University of Adelaide.

SHEPHERD, K. W., AND MAYO, G. M. E. 1972. Genes conferring specific plant disease resistance. Science, $175,375-380$.

SHEPHERD, K. W. AND MAYO, G. M. E. 1979. Studies of genes controlling specific host-parasite interactions in flax and its rust. II. Fine structure analysis of the $L$ group in the host (in prep.).

STEVEns, w. L. 1942. Accuracy of mutation rates. 7. Genetics, 43, 301-307.

WICKs, z. W. III, AND HAMMOND, J. J. 1978. Screening of flax species for new sources of genes resistant to Melampsora lini (Ehrenb.) Lév. Crop Sci., 18, 7-10.

zimMER, D. E. AND COMSTOCK, v. E. 1973. New genes for rust resistance in flax. Phytopath., $63,777-780$. 